\title{
Challenges and Future of Wireless Capsule Endoscopy
}

\author{
Jean-Christophe Saurin, Nicolas Beneche, Christine Chambon and Mathieu Pioche \\ Department of Gastroenterology, E. Herriot Hospital, Hospices Civils de Lyon, Lyon, France
}

In 2015, capsule endoscopy was introduced as the main investigation method for small bowel mucosal diseases, and its role in colonic diseases has been gradually revealed. Future challenges for capsule endoscopy, besides improvements of image quality and visualization of each part of the small bowel and colonic mucosa, include the development of gastric capsules, the capacity to perform histological examination of the mucosa, and maybe in the future, some capsule endoscopy-driven therapeutics. The aim of this review was to evaluate the clinical demands and feasibility of achieving the aforementioned objectives. Clin Endosc 2016;49:26-29

Key Words: Capsule endoscopy; Small bowel; Colon; Stomach; Forecasting

\section{INTRODUCTION}

Capsule endoscopy is an effective, simple, painless, automatic method of imaging the mucosa of the digestive tract that has numerous potential applications and an increasing role in digestive pathological examination. However, multiple improvements of the present capsule endoscopy procedures, adapted to the clinical needs of patients, are possible. The present limitations of the procedure include the following: (1) the time required to read capsule films; (2) the difficulty of identifying some lesions, because of either reader limitations or the inability of the capsule to visualize some lesions (lack of sensitivity); (3) the inability of capsule endoscopy to lead to adequate management of identified lesions through histological examination and precise localization of lesions in the small bowel or colon; (4) limited therapeutic capabilities; and (5) Some attempts have been made to resolve these limitations but are currently still under study.

Received: November 10, 2015 Accepted: November 30, 2015

Correspondence: Jean-Christophe Saurin

Department of Gastroenterology, E. Herriot Hospital, Hospices Civils de Lyon, Pavillon L 2 et. 5 Place d'Arsonval, 69437 Lyon Cedex 03, France

Tel: +33-472-11-0369, Fax: +33-472-11-0147

E-mail: jean-christophe.saurin@chu-lyon.fr

(cc) This is an Open Access article distributed under the terms of the Creative Commons Attribution Non-Commercial License (http://creativecommons.org/ licenses/by-nc/3.0) which permits unrestricted non-commercial use, distribution, and reproduction in any medium, provided the original work is properly cited.

\section{READING TIME AND IDENTIFICATION OF LESIONS}

The sensitivity of capsule endoscopy for the detection of significant lesions relies on the quality of images, number of images the capsule takes in 1 second, and quality of the reading. ${ }^{1-3}$ The quality of images obtained using capsule endoscopy has improved progressively to being comparable with those obtained using conventional endoscopy. The depth of view and luminosity control improves from one generation to another and for every brand of capsule. The number of images seems to be important for assessing sensitivity, but increasing the number of images or using a double-tip capsule, such as the colonic capsule, leads automatically to an important increase in reading time, which is difficult for physicians, especially in countries such as France, where only physicians are allowed to read capsule films. One solution could be a motion sensor, probably because most failures of capsule endoscopy to identify significant lesions are related to rapid transit of the capsule through some digestive segments. Such motion sensor is already used in the colon Pilcam2 capsule (Given Imaging, Yoqneam, Israel) and in the Capsovision small bowel system (CapsoVision, Saratoga, CA, USA), and has been proven to improve the sensitivity of the colon capsule from $64 \%$ to $89 \%$ in recent studies. ${ }^{4,5}$

The future probably lies in automatic algorithms for specific lesion detection. A highly promising algorithm is the 
quick-view reading tool of Given Imaging/Covidien (Dublin, Ireland), which proved to allow rapid reading of small bowel capsule films (mean, 11 minutes), with a $93 \%$ sensitivity for significant lesions. ${ }^{3}$ New algorithms will certainly be developed with a possibility that a computer will propose a series of 5 to 10 diagnoses, from among which the gastroenterologist will simply have to choose the right one. ${ }^{6}$

\section{PRECISE LOCALIZATION OF IDENTIFIED LESIONS}

Only in some cases can capsule endoscopy identify significant imaging findings (e.g., tumors and angiodysplasias) that require treatment or biopsies. This means that at present, a second procedure (balloon-assisted enteroscopy) is necessary. ${ }^{7}$ The main difficulty encountered by teams that perform enteroscopy is the localization of lesions within the small bowel and determining their clinical relevance. ${ }^{8}$ Lesion location is of major importance to determine the enteroscopy route (oral or anal) and, in some cases, to guide surgeons in finding the lesion(s). Indexes have been developed based on the transit time from the pylorus to the lesion and from the lesion to the caecum, but remain of limited utility in clinical practice. ${ }^{9} \mathrm{New}$ devices are under study, but no clear data have been published yet that associate some kind of odometer to an endoscopic capsule. ${ }^{10}$ An informatic algorithm coupled to the capsule film is under evaluation in the Olympus capsule system, but evidence to support its usefulness has not been published yet.

\section{HISTOLOGICAL EXAMINATION}

Methods for performing small bowel biopsies during capsule endoscopy have been proposed. Two technical possibilities have been proposed for obtaining histological evaluation of the small bowel mucosa with a capsule: (1) real samples could be obtained through aspiration/cutting or (2) virtual histological images could be obtained by using confocal imaging or optical coherence tomography. ${ }^{11,12}$ Moreover, two different strategies of biopsies could be used, (1) either random biopsies all along the small bowel at predetermined intervals or (2) targeted biopsies on specific lesions. The former could be useful in patients with known small bowel ulcerations to differentiate; for example, Crohn disease from lymphoma or other inflammatory/infectious diseases; in patients with chronic diarrhea of unknown origin; and for the surveillance of previously treated lymphomas. The latter represents a challenge in terms of medical time because it is impossible for physicians to stay with their patients throughout the duration of the recording of small bowel images and direct biopsies at specific lesions. However, one can imagine automatic detec- tion of significant images, such as an improved "quick view" algorithm, with targeted real/optical biopsies of these specific images. Considering the major usefulness for clinical practice of small bowel biopsies, these kinds of tools will probably be added at some point in time to the arsenal of capsule endoscopy systems. ${ }^{13}$

\section{THERAPEUTICS}

The major advantage of capsule endoscopy of the small bowel is that it can be performed without human intervention, without pain, and with very low risk of complications. In this setting, introducing a treatment tool represents a difficult challenge. As in a science fiction movie, one could imagine a capsule that could coagulate the classic small bowel angiodysplasia, which represents the main diagnosis based on capsule endoscopic results in patients with anemia and occult overt/ obscure bleeding. In order to achieve this, first, capsule endoscopy should be used along with a coagulation tool such as argon plasma coagulation or a type of laser, which would require a great amount of energy and probably a much bigger capsule. Currently, no published study has been conducted on such a tool. However, the main issue would be to direct this kind of treatment during the movement of the capsule to very limited lesions. If we remain in the paradigm of non-human intervention, this necessitates some kind of automatic, highly precise recognition of lesions to be treated. Currently, no informatics algorithm is able to achieve this, although this may change in the future with the improvement of recognition algorithms. However, capsules then would have to be used to treat lesions immediately and in the right direction and location, which appears to be almost impossible at present. For medical intervention in humans, this necessitates that medical personnel stay with their patients for 3 to 7 hours during capsule advancement in order to treat detected lesions under a real-time viewer. Attempts toward this have been made in animal models by using hemostatic clips under human control. ${ }^{13}$ Thus, an efficient and targeted treatment would probably require a capsule controlled from the outside to turn and move it forward and backward. This requires major improvement of capsule control that is far beyond our present capabilities.

\section{SITES OTHER THAN THE COLON AND SMALL BOWEL}

Esophageal capsule endoscopy has been shown to be feasible under specific conditions, including patients in a lateral lying position when swallowing the capsule. The frequently encountered difficulty is the quick passage of the capsule within the cardia, the main site of interest for two different in- 
dications, namely Barrett's esophagus and portal hypertension. This explains its limited sensitivity for Barrett's esophagus of only around $77 \%$ in a published meta-analysis of 618 patients. ${ }^{14}$ The detection rate of epidermoid neoplastic lesions of the esophagus by using capsule endoscopy has been shown to be limited. ${ }^{15}$ New studies that take advantage of the possibility of capsule external control using a magnet would be of interest. This may be a solution for improved examination of the whole esophagus, specifically the lower esophagus.

Gastric examination using capsule endoscopy has been attempted by either simple swallowing or patient mobilization, by using a complex device similar to a magnetic resonance imaging (MRI) system, ${ }^{16}$ and more recently, by using an external magnetic control in healthy volunteers. ${ }^{17}$ However, difficulties are encountered in this setting. First, without specific measures, it is rather difficult to identify all important gastric landmarks, specifically the cardia. In a recent study, a nasogastric tube was inserted to help identify the cardia. Second, spontaneous transit time can be short in some parts of the stomach but long in others parts. External control by using MRI or a simple magnet may improve this situation. Third, the resolution of the capsule images is currently significantly lower than that of typical endoscopic images. This is important, as the main objective of gastric examination in the modern setting is to identify at-risk patients for whom surveillance (for atrophic gastritis and intestinal metaplasia) would be recommended.

The cost of capsule endoscopy will be an important factor regarding the potential development of upper digestive capsule endoscopy. At present, capsule endoscopy is significantly more costly than gastroscopy in some countries such as France. Thus, the balance between sensitivity, quality, simplicity, and cost is highly in favor of conventional endoscopy despite the expected much better patient tolerance and acceptability of capsules.

\section{CAPSULE BEDSIDE ENDOSCOPIC IMAGING}

Evaluating the motility of the small bowel by using capsule endoscopy is an interesting possibility. Different approaches may bring important clinical insight into a relatively unknown area. Clinical indications are rare and specific, such as severe digestive tract motor dysfunction, but the benefits could be significant if therapeutic interventions could be proposed in the future for patients with irritable bowel syndrome. One possibility is the use of the pressure sensors recently developed by Covidien/Given Imaging that can indicate the intensity and site of small bowel contractions. The other possibility is to estimate small bowel motility according to capsule motion and imaging speed, which is possible by using the motion sensor already present in some capsule systems such as Given Imaging/Covidien and CapsoVision., ${ }^{4,18}$ Both approaches are in their preliminary stages, and the results of ongoing studies in different patient populations are awaited in order to make conclusions.

\section{CONCLUSIONS}

Given its availability and simplicity, capsule endoscopy may remain a powerful diagnostic tool for screening and evaluation of numerous pathological conditions, and may progressively replace diagnostic endoscopy. Cost is the main barrier to the rapid development of capsule endoscopy in this setting. We can consider that improved imaging, resolution and lighting, and field of view, and the development of tools for locating small bowel lesions are the more important goals in the development of capsule endoscopy for clinical purposes and generalized use with a diagnostic intent. Histological analysis and treatment driven by findings obtained by using capsule endoscopy represent much more complex challenges. Thus, it may take a long time before capsule endoscopy can become feasible and clinically applicable.

\section{Conflicts of Interest}

The authors have no financial conflicts of interest.

\section{REFERENCES}

1. Saurin JC, Delvaux M, Gaudin JL, et al. Diagnostic value of endoscopic capsule in patients with obscure digestive bleeding: blinded comparison with video push-enteroscopy. Endoscopy 2003;35:576-584.

2. Saurin JC, Delvaux M, Vahedi K, et al. Clinical impact of capsule endoscopy compared to push enteroscopy: 1-year follow-up study. Endoscopy 2005;37:318-323

3. Saurin JC, Lapalus MG, Cholet F, et al. Can we shorten the small-bowel capsule reading time with the "Quick-view" image detection system? Dig Liver Dis 2012;44:477-481.

4. Spada C, Hassan C, Munoz-Navas M, et al. Second-generation colon capsule endoscopy compared with colonoscopy. Gastrointest Endosc 2011;74:581-589.e1.

5. Van Gossum A, Munoz-Navas M, Fernandez-Urien I, et al. Capsule endoscopy versus colonoscopy for the detection of polyps and cancer. $\mathrm{N}$ Engl J Med 2009;361:264-270.

6. Subramanian V, Mannath J, Telakis E, Ragunath K, Hawkey CJ. Efficacy of new playback functions at reducing small-bowel wireless capsule endoscopy reading times. Dig Dis Sci 2012;57:1624-1628.

7. May A, Friesing-Sosnik T, Manner H, Pohl J, Ell C. Long-term outcome after argon plasma coagulation of small-bowel lesions using double-balloon enteroscopy in patients with mid-gastrointestinal bleeding. Endoscopy 2011;43:759-765.

8. Li X, Chen H, Dai J, Gao Y, Ge Z. Predictive role of capsule endoscopy on the insertion route of double-balloon enteroscopy. Endoscopy 2009;41:762-766.

9. Gay G, Delvaux M, Fassler I. Outcome of capsule endoscopy in determining indication and route for push-and-pull enteroscopy. Endoscopy 
2006;38:49-58.

10. Karargyris A, Koulaouzidis A. Capsule-odometer: a concept to improve accurate lesion localisation. World J Gastroenterol 2013;19:5943-5946.

11. Gora MJ, Sauk JS, Carruth RW, et al. Tethered capsule endomicroscopy enables less invasive imaging of gastrointestinal tract microstructure. Nat Med 2013;19:238-240.

12. Sauk J, Coron E, Kava L, et al. Interobserver agreement for the detection of Barrett's esophagus with optical frequency domain imaging. Dig Dis Sci 2013;58:2261-2265.

13. Valdastri P, Quaglia C, Susilo E, et al. Wireless therapeutic endoscopic capsule: in vivo experiment. Endoscopy 2008;40:979-982.

14. Bhardwaj A, Hollenbeak CS, Pooran N, Mathew A. A meta-analysis of the diagnostic accuracy of esophageal capsule endoscopy for Barrett's esophagus in patients with gastroesophageal reflux disease. Am J Gastroenterol 2009;104:1533-1539.
15. Heresbach D, Leray E, d'Halluin PN, et al. Diagnostic accuracy of esophageal capsule endoscopy versus conventional upper digestive endoscopy for suspected esophageal squamous cell carcinoma. Endoscopy 2010;42:93-97.

16. Denzer UW, Rösch T, Hoytat B, et al. Magnetically guided capsule versus conventional gastroscopy for upper abdominal complaints: a prospective blinded study. J Clin Gastroenterol 2015;49:101-107.

17. Rahman I, Pioche M, Shim CS, et al. Magnet assisted capsule endoscopy (MACE) in the upper GI tract is feasible: first human series using the novel Mirocam-Navi system. Gastrointest Endosc 2014;79(5 Sup$\mathrm{pl}): \mathrm{AB} 22$.

18. Pioche M, Vanbiervliet G, Jacob P, et al. Prospective randomized comparison between axial- and lateral-viewing capsule endoscopy systems in patients with obscure digestive bleeding. Endoscopy 2014;46:479-484. 